\title{
Modified Bezier Curve for 4D Reference Trajectory Definition under Flight Profile Constraint
}

\author{
Thierry Miquel* \\ Direction des Services de la Navigation Aérienne, Toulouse, FRANCE \\ Félix Mora-Camino ${ }^{\dagger}$ \\ Ecole Nationale de l'Aviation Civile and LAAS du CNRS, Toulouse, France
}

\begin{abstract}
The task of establishing properly spaced landing sequences is quite demanding for air traffic controllers in heavy traffic conditions. Indeed, air traffic controllers combine two or more streams into a single stream before landing by means of radar vectoring and speed instructions. This high level task of sequencing aircraft is not currently communicated to the pilot. Instead, controller translate it into clearances to the pilot, typically radar vectoring and speed instructions. In this paper, the task of merging an aircraft over a specified meter fix is addressed through a novel 'Set Reference Path' procedure in which the air traffic controller may clear an aircraft to merge at a specified meter fix at a given time. This paper focuses on the definition of a reference path for time-based operations at meter fix. It includes path stretching operations, which occur when the aircraft is predicted to overfly too early a meter fix with respect to other traffic flow constraints. This paper presents a new approach to generate a reference path with length and endpoint constraints based on modified Bezier curves. Compared to reference trajectories based on straight lines and circle arcs, which are usual in the aviation community, the proposed approach allows for smooth control cues and avoids the scheduling between straight lines and circle arcs segments. The proposed design is followed by illustrative examples which show the effectiveness of the proposed approach.
\end{abstract}

\section{Introduction}

$\mathrm{F}$ rom the ICAO definition ${ }^{1}$, ATS services include the prevention of collisions between aircraft and between aircraft on the ground and obstructions, the provision of advice to aircraft and information required for the safe and efficient conduct of flights and the achievement of an orderly and expeditious flow of air traffic. The overall goal of the R\&D community has always been to improve Air Traffic Services (ATS) through a greater involvement of pilots in cooperation with air traffic controllers. For example, the Airborne Separation Assistance System (ASAS) concept was presented to ICAO in 2003 to take advantage of new airborne capabilities for surveillance ${ }^{2}$. Indeed, ASAS is an airborne system that allows air traffic controllers to possibly delegate separation responsibility and transfers the corresponding separation tasks to the flight crew. ASAS allows the flight crew to maintain separation between their aircraft and one or more other aircraft, and provides information concerning the surrounding traffic. This system and associated concept basically address the ATS services dealing with the prevention of collisions and the provision of information on surrounding traffic to aircraft.

This paper addresses another aspect of ATS, namely the achievement of an orderly and expeditious flow of air traffic. Indeed, the task of establishing properly spaced landing sequences is quite demanding for air traffic controllers in heavy traffic conditions. More specifically, air traffic controllers combine two or more streams into a single stream before landing by means of radar vectoring and speed instructions. This high level task of sequencing aircraft is not currently communicated to the pilot. Instead, controller translate it into clearances to the pilot, typically radar vectoring and speed instructions. In this paper, the task of merging an aircraft over a specified meter fix is addressed through a novel 'Set Reference Path' procedure in which the air traffic controller may clear an

\footnotetext{
* PhD, Civil Aviation Design and Operation Engineer, Separation and Alerting Systems Unit, miquel@cena.fr $\dagger$ Senior Researcher at LAAS du CNRS, Professor of Automatic Control and Avionics at Ecole Nationale de l'Aviation civile, Felix.MORA@enac.fr Copyright $@ 2008$ by DSNA. Published by the American Institute of Aeronautics and Astronautics, Inc., with permission.
} 
aircraft to merge at a specified meter fix at a given time. This procedure may be envisioned as the airborne counterpart of the ground based Arrival MANager (AMAN), which is a tactical controller assistance system enabling the computation of rendez-vous time at meter fix to meet the runway capacity and absorb the traffic. The time computed by the AMAN may then be used by the air traffic controller to ask the aircrew to overfly the meter fix at the desired time. This type of procedure is clearly in the scope of the 4D trajectory concept promoted by the European project SESAR ${ }^{3}$.

While keeping the controller in charge of Air Traffic Management (ATM), the procedure involves new avionics capabilities for merging operations, including path definition capabilities as well as tracking altitude, speed and heading cues. Indeed controllers remain responsible for making the traffic flow decisions; the change is that the controller would then communicate these decisions at a higher level to the pilot, e.g. 'Be at Meter Fix ABCDE at 13:20:35' rather than requiring the controller to calculate and communicate lower-level guidance instructions. The main expected benefit of this application is to increase capacity through the decrease of controller's workload and to improve flight efficiency by more precise maneuvering from onboard capabilities.

As already mentioned, air traffic controller keeps the task of preventing collisions between aircraft. Thus, this kind of procedure is not an ASAS procedure. Nevertheless, it is also envisioned to combine this procedure with ASAS target trail mode in order to render possible a trailing aircraft to track the delayed path of the 4D managed aircraft, and thus maintain spacing between both aircraft, as showed in the following figure:

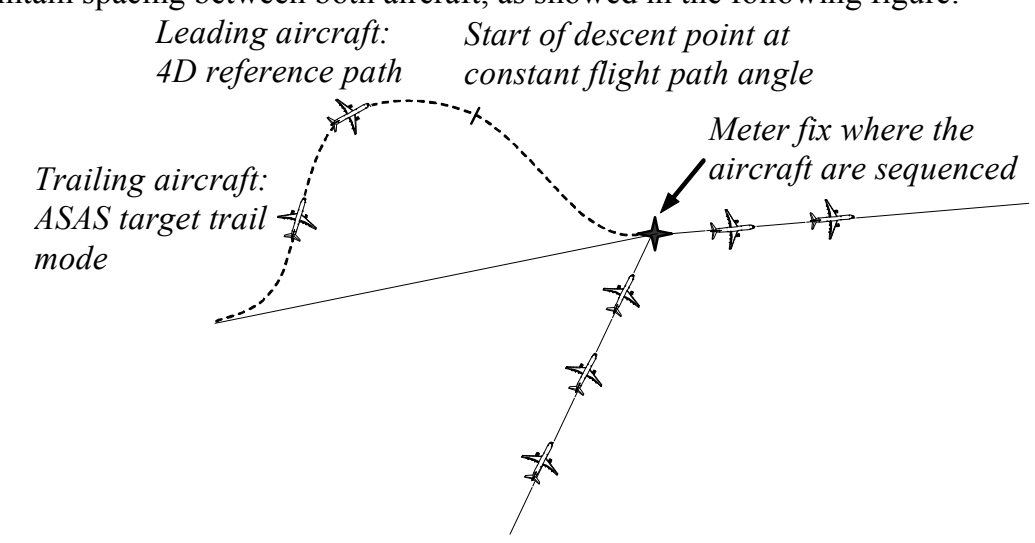

Fig. 1: combined ASAS target trail and 4D reference path operations

This paper focuses on the definition of a reference path for time-based operations at meter fix. It includes path stretching operations, which occur when the aircraft is predicted to overfly a meter fix with too early respect to other traffic flow constraints. The reference path definition problem consists in finding curves that satisfy constraints on length and on the positions and tangents at the two endpoints. Finding a smooth path with continuous curvature that can match arbitrary endpoint constraints and length constraint is nontrivial. Several techniques can be used, including geometric approaches ${ }^{4,5,6}$, the use of optimal control ${ }^{7,8}$ as well as evolutionary algorithms ${ }^{9}$. In this paper, a geometric approach using modified Bezier curves is introduced.

The paper is organized as follows: in the next section, the parameterization of the reference trajectory is addressed. Then the definition of the reference trajectory for an airliner in order to obtain its arrival on a meter fix at a time chosen by air traffic control is addressed. This is done on the basis of modified Bezier curves which allows generating a path with length and endpoint constraints. The reference trajectory definition is followed by illustrative examples where level changes and wind are considered. Finally, conclusions on the effectiveness of the proposed approach are drawn.

\section{Reference trajectory parameterization}

\section{A. Assumptions on flight profile}

Most of Flight Crew Operating Manuals do not provide operating speed in terms of true airspeed, denoted $V$ hereafter, but in terms of calibrated airspeed or Mach number. It is assumed in the following that the equivalent airspeed $V_{e}$, that is the calibrated airspeed corrected for the scale altitude error, is controlled by the aircrew. Denoting by $\sigma(h)$ the relative air density, which depends on altitude $h$, the relationship between true airspeed $V$ and equivalent airspeed $V_{e}$ is the following ${ }^{10}$ : 


$$
\left\{\begin{array} { l } 
{ V = \frac { V _ { e } } { \sqrt { \sigma ( h ) } } } \\
{ \sigma ( h ) = \frac { \rho ( h ) } { \rho ( 0 ) } }
\end{array} \text { where } \left\{\begin{array}{l}
\sqrt{\sigma(h)}=(1+b \cdot h)^{-\lambda} \\
-\lambda=-\frac{1}{2} \cdot\left(\frac{g}{k_{T} \cdot R}+1\right) \approx \frac{4.2558}{2} \\
b=\frac{k_{T}}{T_{0}} \approx-\frac{0.0065}{288.15} m^{-1}
\end{array}\right.\right.
$$

As far as the flight profile is concerned, it is assumed in the following that the 4D maneuver starts at flight level $h_{0}$ and is achieved at flight level $h_{l}$. Flight level phase at $h_{0}$ is assumed to be followed by a descent phase at constant flight path angle, denoted $\gamma_{d}$, until flight level $h_{l}$ is reached. Furthermore it is assumed that the equivalent airspeed of the aircraft is constant and maintained to $V_{e 0}$ while the aircraft is level. Then the equivalent airspeed is assumed to linearly decrease towards $V_{e l}$ during the descent phase. The following figure depicts the assumptions on the flight profile, where $t_{d}$ denotes the duration of the descent phase, $t_{s}$ the duration of the slow down phase where the equivalent airspeed decreases from $V_{e 0}$ to $V_{e l}$. In addition $T$ denotes the requested instant at which the aircraft should be over the meter fix specified by the air traffic controller:

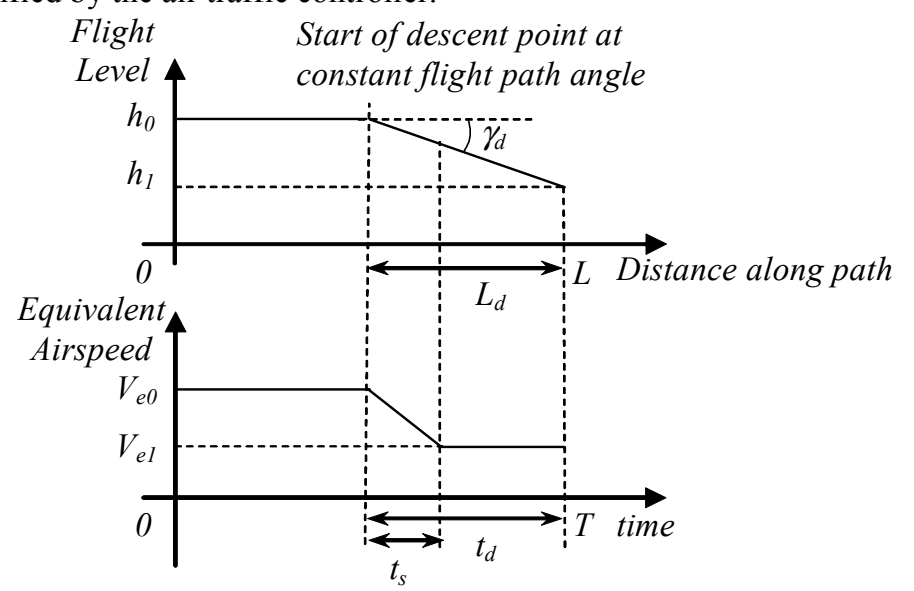

Fig. 2: assumption on flight profile

The rationale to assume a constant flight path angle during the descent comes from the longitudinal equation of motion. This equation is derived from Newton's second law of motion ${ }^{11}$, where $F$ is the thrust of the aircraft, $D$ the drag, $m$ the mass and $g$ the gravitational acceleration:

$$
m \cdot \frac{d V}{d t}=(F-D)-m g \cdot \sin (\gamma)
$$

Introducing (1) and its time derivative in (2), we get:

$$
\sin (\gamma)=\left(\frac{F-D}{m g}-\frac{\dot{V}_{e}}{g \cdot \sqrt{\sigma(h)}}\right) \cdot \frac{1}{(1-f(h))} \text { where } f(h)=V_{e}^{2} \cdot \frac{d \sigma(h) / d h}{2 \cdot g \cdot \sigma^{2}(h)}
$$

Calculations show that term $f(h)$ can be neglected compared to $l$ when equivalent airspeed $V_{e}$ and flight level $h$ are set to typical values observed in approach control. Indeed, when flight level $h$ varies from 0 to FL100 and for two values of equivalent airspeed, $100 \mathrm{kts}$ and $250 \mathrm{kts}$, it can be seen that the maximum value of $f(h)$ is achieved at $250 \mathrm{kts}$ and $F L 100$, where the term $f(h)$ is about -0.117 .

Consequently, assuming that acceleration of equivalent airspeed is small compared to gravitational acceleration $g$ and that aircraft mass $m$ and difference between thrust $F$ and drag $D$ remain constant, flight path angle $\gamma$ can be assumed to remain constant during approach control.

Assuming that the requested duration $T$ of the trajectory is known, the purpose of the following development is to express the value of duration $t_{d}$ which enables to locate the start of descent instant and the value of the requested length $L$ of the trajectory.

First, during the descent phase and duration $t_{s}$ (see Fig. 2), the equivalent airspeed is assumed to have the following expression:

$$
V_{e}(t)=V_{e 0}+\frac{t}{t_{s}} \cdot\left(V_{e 1}-V_{e 0}\right)
$$

In addition, the vertical speed of the aircraft is given by: 


$$
\frac{d h}{d t}=V(t) \cdot \sin \left(\gamma_{d}\right)
$$

Combining equations (1), (4) and (5) lead to the following nonlinear first order differential equation:

$$
\sqrt{\sigma(h)} \cdot d h=\left(V_{e 0}+\frac{t}{t_{s}} \cdot\left(V_{e 1}-V_{e 0}\right)\right) \cdot \sin \left(\gamma_{d}\right) \cdot d t
$$

Solving this differential equation under the assumption of standard atmosphere model ${ }^{11}$ (below the Tropopause) leads to the expression of flight level $h$ as a function of time $t$ between instants $t_{d}$ and $t_{d}+t_{s}$ :

$$
\frac{(1+b \cdot h(t))^{1-\lambda}-\left(1+b \cdot h_{0}\right)^{1-\lambda}}{b \cdot(1-\lambda)}=V_{e 0} \cdot t \cdot \sin \left(\gamma_{d}\right) \cdot\left(1+\frac{t}{2 \cdot t_{s}} \cdot\left(\frac{V_{e l}}{V_{e 0}}-1\right)\right)
$$

We then get the value of flight level $h\left(t_{s}\right)$ :

$$
h\left(t_{s}\right)=\frac{1}{b} \cdot\left(\left(\left(1+b \cdot h_{0}\right)^{1-\lambda}+V_{e 0} \cdot t_{s} \cdot \sin \left(\gamma_{d}\right) \cdot b \cdot(1-\lambda) \cdot\left(1+\frac{1}{2} \cdot\left(\frac{V_{e 1}}{V_{e 0}}-1\right)\right)\right)^{\frac{1}{1-\lambda}}-1\right)
$$

In addition, the time needed to go from flight level $h\left(t_{s}\right)$ to $h_{1}$ can be evaluated by an expression similar to (7) where $V_{e 0}$ is replaced by $V_{e l}$ as far as during that segment the equivalent airspeed is constant and set to $V_{e l}$ :

$$
\frac{\left(1+b \cdot h_{1}\right)^{1-\lambda}-\left(1+b \cdot h\left(t_{s}\right)\right)^{1-\lambda}}{b \cdot(1-\lambda)}=V_{e 1} \cdot\left(t_{d}-t_{s}\right) \cdot \sin \left(\gamma_{d}\right)
$$

Assuming that deceleration duration $t_{s}$, flight levels $h_{0}$ and $h_{1}$, equivalent airspeed $V_{e 0}$ and $V_{e l}$ are known, we get from the preceding equation the value of descent duration $t_{d}$ as a function of $h\left(t_{s}\right)$, which is available through (8):

$$
t_{d}=t_{s}+\frac{1}{V_{e 1} \cdot \sin \left(\gamma_{d}\right)} \cdot\left(\frac{\left(1+b \cdot h_{1}\right)^{1-\lambda}-\left(1+b \cdot h\left(t_{s}\right)\right)^{1-\lambda}}{b \cdot(1-\lambda)}\right)
$$

Finally, assuming that the requested duration of flight $T$ is known and greater than duration of the descent phase $t_{d}$, the required length $L$ of the trajectory is found to be:

$$
L=V\left(h_{0}\right) \cdot\left(T-t_{d}\right)+L_{d}
$$

Where the distance $L_{d}$ which is flown during the descent phase has the following expression:

$$
L_{d}=\left|\frac{h_{1}-h_{0}}{\sin \left(\gamma_{d}\right)}\right|
$$

\section{B. Trajectory parameterization}

Assuming that Earth is flat and non-rotating, it may be considered as an inertial frame. The kinematics equations describing the aircraft motion are recalled hereafter, where $x, y$ and $h$ refer to Euclidian position, no subscript refers to the airframe and subscript $g$ to earth reference frame. In the following, $V$ denotes the airspeed of the aircraft, $\gamma$ its flight path angle and $\psi$ its heading. In addition $\psi_{w}$ denotes the direction from where the (horizontal) wind is blowing and $V_{w}$ the constant wind velocity, and no vertical wind component is assumed:

$$
\left\{\begin{array}{l}
\dot{x}_{g}(t)=\dot{x}(t)+V_{w} \cdot \cos \left(\psi_{w}-\pi\right) \\
\dot{y}_{g}(t)=\dot{y}(t)+V_{w} \cdot \sin \left(\psi_{w}-\pi\right) \\
\dot{h}_{g}(t)=\dot{h}(t)
\end{array}\right.
$$

Where

$$
\left\{\begin{array}{l}
\dot{x}(t)=V(t) \cdot \cos (\gamma(t)) \cdot \cos (\psi(t)) \\
\dot{y}(t)=V(t) \cdot \cos (\gamma(t)) \cdot \sin (\psi(t)) \\
\dot{h}(t)=V(t) \cdot \sin (\gamma(t))
\end{array}\right.
$$

Integrating the preceding set of equations leads to expression of the ground based aircraft coordinates $x_{g}(t), y_{g}(t)$ and $h_{g}(t)$ as a function of the air based aircraft coordinates $x(t), y(t)$ and $h(t)$ : 


$$
\left\{\begin{array} { l } 
{ x _ { g } ( t ) = x _ { g } ( 0 ) + x ( t ) + V _ { w } \cdot t \cdot \operatorname { c o s } ( \psi _ { w } - \pi ) } \\
{ y _ { g } ( t ) = y _ { g } ( 0 ) + y ( t ) + V _ { w } \cdot t \cdot \operatorname { s i n } ( \psi _ { w } - \pi ) } \\
{ h _ { g } ( t ) = h _ { g } ( 0 ) + h ( t ) }
\end{array} \quad \text { where } \left\{\begin{array}{l}
x(t)=\int_{0}^{t} \dot{x}(u) \cdot d u \\
y(t)=\int_{0}^{t} \dot{y}(u) \cdot d u \\
h(t)=\int_{0}^{t} \dot{h}(u) \cdot d u
\end{array}\right.\right.
$$

As described in the introduction section, the envisioned operational procedure assumes that the air traffic control requests the aircrew to be over a given meter fix at a given time $T$ and at the given flight level $h_{l}$. The aircrew will comply with that request by adjusting the length of the trajectory under constrained flight level and equivalent airspeed. Indeed, it is assumed that airspeed of the aircraft is indirectly known and imposed for example by Flight Crew Operating Manual. So, the reference trajectory definition problem consists in finding a mapping $t \rightarrow \mathbf{P}(t)=[x(t), y(t), h(t)]^{T}$ such that constraints on the positions and tangents at the two endpoints at time $t=0$ and $t=T$ are satisfied. Furthermore, the mapping $t \rightarrow \mathbf{P}(t)=[x(t), y(t), h(t)]^{T}$ shall also satisfy speed constraints.

In the following the aircraft trajectory is described as mapping from a dimensionless interval to $R^{3}$. More precisely, let $\mathbf{P}(\tau) \rightarrow R^{3}$ be the aircraft trajectory, where dimensionless parameter $\tau$ is taken within the interval $[0,1]$. The definition of dimensionless parameter $\tau$ may either involve time or arc length. In order to alleviate the mapping to be found from the speed constraint, parameter $\tau$ has be chosen to be proportional to the arc length $s(t)$ but not to time $t$. Consequently, the constraint on speed profile is changed into a constraint on the length of the trajectory. More specifically, dimensionless parameter $\tau$ is defined hereafter where $T$ is the prescribed duration of the maneuver and $L$ the length of the trajectory when the maneuver is completed:

$$
\tau(t)=\frac{s(t)}{L} \text { where }\left\{\begin{array}{l}
s(0) \hat{=} 0 \\
s(T) \hat{=} L
\end{array}\right.
$$

The rationale for this parameterization comes from the definition of the airspeed which is the time derivative of the arc length, that is:

$$
V(t)=\frac{d s(t)}{d t}
$$

In addition, the time derivative of parameter $\tau$ can be deduced from (16) and (17) by using the derivative chain rule:

$$
\frac{d \tau(t)}{d t}=\frac{d \tau(s)}{d s} \cdot \frac{d s(t)}{d t}=\frac{V(t)}{L}
$$

When using the previous relationship with (14), we get:

$$
\left\{\begin{array}{l}
\frac{d}{d \tau} x(\tau)=L \cdot \cos (\gamma(\tau)) \cdot \cos (\psi(\tau)) \\
\frac{d}{d \tau} y(\tau)=L \cdot \cos (\gamma(\tau)) \cdot \sin (\psi(\tau)) \\
\frac{d}{d \tau} h(\tau)=L \cdot \sin (\gamma(\tau))
\end{array}\right.
$$

It is worth noticing that airspeed $V(t)$ simplifies in the previous equations. Consequently trajectory $\mathbf{P}(\tau)=[x(\tau), y(\tau), h(\tau)]^{T}$ is now independent from airspeed: this justifies to choose parameter $\tau$ proportional to the arc length. The two endpoints of the trajectory are denoted respectively $\mathbf{P}(0)$ and $\mathbf{P}(1)$, where $\mathbf{P}(0)$ is the starting point of the trajectory and $\mathbf{P}(1)$ is the ending point. The Cartesian expression of trajectory $\mathbf{P}(\tau)$ is:

$$
\mathbf{P}(\tau)=\left[\begin{array}{l}
x(\tau) \\
y(\tau) \\
h(\tau)
\end{array}\right]
$$

As far as initial and final flight path angle and heading are assumed to be known, trajectory $\mathbf{P}(\tau)$ shall satisfy the following endpoint constraints, where $\gamma_{d}$ stands for the final flight path angle and $\psi_{0}$ and $\psi_{1}$ stand for the initial and final heading: 


$$
\left\{\begin{array} { l } 
{ \frac { d } { d \tau } x ( 0 ) = L \cdot \operatorname { c o s } ( \psi _ { 0 } ) } \\
{ \frac { d } { d \tau } y ( 0 ) = L \cdot \operatorname { s i n } ( \psi _ { 0 } ) \quad \text { where } } \\
{ \frac { d } { d \tau } h ( 0 ) = 0 }
\end{array} \left\{\begin{array}{l}
\frac{d}{d \tau} x(1)=L \cdot \cos \left(\psi_{1}\right) \cdot \cos \left(\gamma_{d}\right) \\
\frac{d}{d \tau} y(1)=L \cdot \sin \left(\psi_{1}\right) \cdot \cos \left(\gamma_{d}\right) \\
\frac{d}{d \tau} h(1)=L \cdot \sin \left(\gamma_{d}\right)
\end{array}\right.\right.
$$

In addition, flight level $h(\tau)$ is set as follows where $\Gamma(x)$ stands for the Heaviside step function (its value is 1 if $x>0$, and 0 otherwise) in order to satisfy the requested vertical flight profile:

$$
h(\tau)=h_{0}+\left(h_{1}-h_{0}\right) \cdot\left(\frac{\tau-\tau_{d}}{1-\tau_{d}}\right) \cdot \Gamma\left(\tau-\tau_{d}\right) \quad \text { where } \quad \tau_{d}=1-\left|\frac{h_{1}-h_{0}}{L \cdot \sin \left(\gamma_{d}\right)}\right|
$$

The preceding relationship completely defines the vertical profile of the aircraft. The purpose of the next section is to define the lateral reference trajectory of the aircraft, that is the expression of horizontal coordinates $x(\tau)$ and $y(\tau)$.

\section{Reference trajectory Definition}

\section{A. Bezier curve}

The objective of the reference trajectory definition consists of generating a smooth trajectory by joining two distinct configurations, the oriented starting and ending points, with a constraint on the length at which the ending point is reached. In this section, vector $[x(\tau), y(\tau)]^{T}$ is assumed to follow a Bezier curve of dimension 2:

$$
\left[\begin{array}{l}
x(\tau) \\
y(\tau)
\end{array}\right]=\sum_{i=0}^{3}\left(\begin{array}{l}
3 \\
i
\end{array}\right) \cdot \tau^{i} \cdot(1-\tau)^{3-i} \cdot \mathbf{P}_{i / 3}
$$

Points $\mathbf{P}_{0 / 3}$ and $\mathbf{P}_{3 / 3}$ are respectively the (given) starting and ending points of the trajectory. Points $\mathbf{P}_{\mathrm{i} / 3}, \mathrm{i} \in\{1,2\}$, are used as parameterization points of the trajectory. Points $\mathbf{P}_{1 / 3}$ and $\mathbf{P}_{2 / 3}$ are used to direct the initial and final orientation of the trajectory according to the initial and final headings. The following figure presents a possible positioning of the parameterization points $\mathbf{P}_{\mathrm{i} / 3}$, where $\psi_{0}$ and $\psi_{1}$ stands for the initial and final heading, and where $\psi_{2}$ stands for the direction of the vector $\mathbf{P}_{0 / 3} \mathbf{P}_{3 / 3}$ :

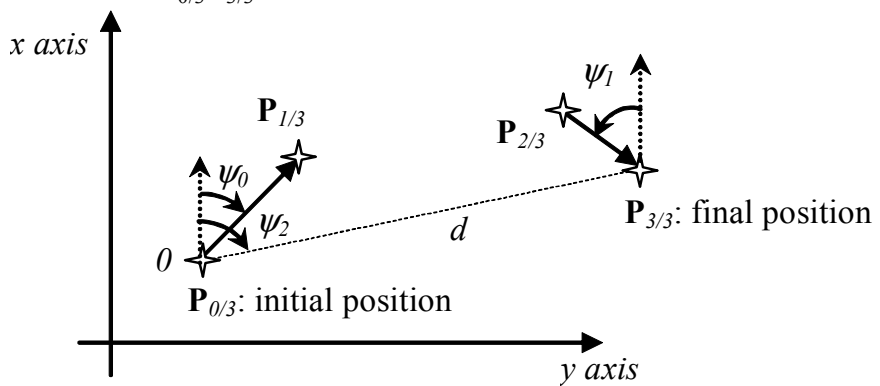

Fig. 3: Parameterization of Bezier curve

Without loss of generality, points $\mathbf{P}_{0 / 3}$ and $\mathbf{P}_{3 / 3}$ are set as follows, where $d$ is the distance between initial and final positions:

$$
\left\{\begin{array}{l}
\mathbf{P}_{0 / 3} \hat{=}\left[\begin{array}{l}
0 \\
0
\end{array}\right] \\
\mathbf{P}_{3 / 3}-\mathbf{P}_{0 / 3} \hat{=} d \cdot\left[\begin{array}{l}
\cos \left(\psi_{2}\right) \\
\sin \left(\psi_{2}\right)
\end{array}\right]
\end{array}\right.
$$

Then, the first derivative of vector $[x(\tau), y(\tau)]^{T}$ is given by:

$$
\frac{d}{d \tau}\left[\begin{array}{l}
x(\tau) \\
y(\tau)
\end{array}\right]=3 \cdot(\tau-1) \cdot(3 \tau-1) \cdot \mathbf{P}_{1 / 3}+3 \cdot \tau \cdot(2-3 \tau) \cdot \mathbf{P}_{2 / 3}+3 \cdot \tau^{2} \cdot d \cdot\left[\begin{array}{c}
\cos \left(\psi_{2}\right) \\
\sin \left(\psi_{2}\right)
\end{array}\right]
$$

If we set points static $\mathbf{P}_{1 / 3}$ and $\mathbf{P}_{2 / 3}$ such that endpoint constraints (21) are satisfied, it can be seen that no degree of freedom remains to satisfy the constraint that the length of the trajectory shall be equal to $L$. In order to alleviate this issue, points $\mathbf{P}_{1 / 3}$ and $\mathbf{P}_{2 / 3}$ have been defined as a function of parameter $\tau$. 


\section{B. Modified Bezier curve}

Compared to classical Bezier curve where points $\mathbf{P}_{\mathrm{i} / 3}, \mathrm{i} \in\{0,1,2,3\}$ do not evolve with parameter $\tau$, a new approach is developed in this paper where points $\mathbf{P}_{1 / 3}$ and $\mathbf{P}_{2 / 3}$ are a function of parameter $\tau$. First, relationship (23) is expanded as follows:

$$
\left[\begin{array}{l}
x(\tau) \\
y(\tau)
\end{array}\right]=(1-\tau)^{3} \cdot \mathbf{P}_{0 / 3}+3 \cdot \tau \cdot(1-\tau)^{2} \cdot \mathbf{P}_{1 / 3}(\tau)+3 \cdot \tau^{2} \cdot(1-\tau) \cdot \mathbf{P}_{2 / 3}(\tau)+\tau^{3} \cdot \mathbf{P}_{3 / 3}
$$

Then, the following parameterization of points $\mathbf{P}_{1 / 3}$ and $\mathbf{P}_{2 / 3}$ is introduced, where $d$ is the Euclidian distance between starting point $\mathbf{P}_{0 / 3}$ and final point $\mathbf{P}_{3 / 3}, L$ is the desired length of the trajectory and where $\lambda_{0}$ and $\lambda_{1}$ are constant parameters to be set:

$$
\left\{\begin{array}{l}
\mathbf{P}_{1 / 3}(\tau) \hat{=} \mathbf{P}_{0 / 3}+\left(\lambda_{0} \cdot \tau+\frac{1}{3}\right) \cdot L \cdot\left[\begin{array}{l}
\cos \left(\psi_{0}\right) \\
\sin \left(\psi_{0}\right)
\end{array}\right] \\
\mathbf{P}_{2 / 3}(\tau) \hat{=} \mathbf{P}_{3 / 3}+\left(\lambda_{1} \cdot(\tau-1)-\frac{1}{3}\right) \cdot L \cdot\left[\begin{array}{l}
\cos \left(\psi_{1}\right) \cdot \cos \left(\psi_{d}\right) \\
\sin \left(\psi_{1}\right) \cdot \cos \left(\gamma_{d}\right)
\end{array}\right]
\end{array}\right.
$$

Using parameterization (27) into the general expression of modified Bezier curve (26) leads to the following expression:

$$
\left[\begin{array}{l}
x(\tau) \\
y(\tau)
\end{array}\right]=3 \cdot \tau \cdot(1-\tau)^{2} \cdot \mathbf{P}_{1 / 3}(\tau)+3 \cdot \tau^{2} \cdot(1-\tau) \cdot \mathbf{P}_{2 / 3}(\tau)+\tau^{3} \cdot d \cdot\left[\begin{array}{c}
\cos \left(\psi_{2}\right) \\
\sin \left(\psi_{2}\right)
\end{array}\right]
$$

The first and second derivative of $\mathrm{x}(\tau)$ and $\mathrm{y}(\tau)$ are the following:

$$
\begin{array}{r}
\frac{d}{d \tau}\left[\begin{array}{l}
x(\tau) \\
y(\tau)
\end{array}\right]=3 \cdot(\tau-1) \cdot(3 \tau-1) \cdot \mathbf{P}_{1 / 3}(\tau)+3 \cdot \tau \cdot(2-3 \tau) \cdot \mathbf{P}_{2 / 3}(\tau)+3 \cdot \tau^{2} \cdot d \cdot\left[\begin{array}{l}
\cos \left(\psi_{2}\right) \\
\sin \left(\psi_{2}\right)
\end{array}\right] \\
+3 \cdot \tau \cdot(1-\tau)^{2} \cdot \lambda_{0} \cdot L \cdot\left[\begin{array}{l}
\cos \left(\psi_{0}\right) \\
\sin \left(\psi_{0}\right)
\end{array}\right]+3 \cdot \tau^{2} \cdot(1-\tau) \cdot \lambda_{1} \cdot L \cdot\left[\begin{array}{l}
\cos \left(\psi_{1}\right) \cdot \cos \left(\gamma_{d}\right) \\
\sin \left(\psi_{1}\right) \cdot \cos \left(\gamma_{d}\right)
\end{array}\right] \\
\frac{d^{2}}{d \tau^{2}}\left[\begin{array}{l}
x(\tau) \\
y(\tau)
\end{array}\right]=3 \cdot(6 \tau-4) \cdot \mathbf{P}_{1 / 3}(\tau)+3 \cdot(2-6 \tau) \cdot \mathbf{P}_{2 / 3}(\tau)+6 \cdot \tau \cdot d \cdot\left[\begin{array}{l}
\cos \left(\psi_{2}\right) \\
\sin \left(\psi_{2}\right)
\end{array}\right] \\
+3 \cdot(\tau-1) \cdot(3 \tau-1) \cdot \lambda_{0} \cdot L \cdot\left[\begin{array}{l}
\cos \left(\psi_{0}\right) \\
\sin \left(\psi_{0}\right)
\end{array}\right]+3 \cdot \tau \cdot(2-3 \tau) \cdot \lambda_{1} \cdot L \cdot\left[\begin{array}{l}
\cos \left(\psi_{1}\right) \cdot \cos \left(\gamma_{d}\right) \\
\sin \left(\psi_{1}\right) \cdot \cos \left(\gamma_{d}\right)
\end{array}\right]
\end{array}
$$
(21):

We get the following values at the beginning and at the end of the trajectory, which satisfy endpoint constraints

$$
\begin{gathered}
{\left[\begin{array}{l}
x(0) \\
y(0)
\end{array}\right]=\left[\begin{array}{l}
0 \\
0
\end{array}\right] \text { and }\left[\begin{array}{l}
x(1) \\
y(1)
\end{array}\right]=d \cdot\left[\begin{array}{l}
\cos \left(\psi_{2}\right) \\
\sin \left(\psi_{2}\right)
\end{array}\right]} \\
{\left[\begin{array}{l}
\frac{d x}{d \tau}(0) \\
\frac{d y}{d \tau}(0)
\end{array}\right]=3 \cdot \mathbf{P}_{1 / 3}(0)=L \cdot\left[\begin{array}{l}
\cos \left(\psi_{0}\right) \\
\sin \left(\psi_{0}\right)
\end{array}\right] \text { and }\left[\begin{array}{l}
\frac{d x}{d \tau}(1) \\
\frac{d y}{d \tau}(1)
\end{array}\right]=-3 \cdot \mathbf{P}_{2 / 3}(1)+3 \cdot d \cdot\left[\begin{array}{l}
\cos \left(\psi_{2}\right) \\
\sin \left(\psi_{2}\right)
\end{array}\right]=L \cdot\left[\begin{array}{l}
\cos \left(\psi_{1}\right) \cdot \cos \left(\gamma_{d}\right) \\
\sin \left(\psi_{1}\right) \cdot \cos \left(\gamma_{d}\right)
\end{array}\right]}
\end{gathered}
$$

\section{Optimization problem}

Now that a generic shape of the trajectory has been defined, parameters $\lambda_{0}$ and $\lambda_{1}$ have to be set to satisfy length constraint. As far as we have two parameters to be set, parameters $\lambda_{0}$ and $\lambda_{l}$ will be set to satisfy the length trajectory constraint while minimizing the mean square of curvature of the trajectory. The rationale for the minimization of the mean square of curvature is to provide as best as possible a flyable trajectory.

The length $l$ of the trajectory when parameter $\tau$ varies from 0 to 1 is defined as a function of parameters $\lambda_{0}$ and $\lambda_{1}$ by:

$$
l\left(\lambda_{0}, \lambda_{1}\right)=\int_{0}^{1} \sqrt{\frac{d \mathbf{P}(\tau)^{T}}{d \tau} \cdot \frac{d \mathbf{P}(\tau)}{d \tau}} \cdot d \tau
$$

The curvature $K(s)$ of the trajectory $\mathbf{P}(\tau)$ is defined by the following relationship ${ }^{12}$, where $s$ represents the arc length of the trajectory:

$$
K(s)=\sqrt{\left(\frac{d^{2} \mathbf{P}(s)}{d s^{2}}\right)^{T} \cdot \frac{d^{2} \mathbf{P}(s)}{d s^{2}}}
$$


In the following, $k$ is used to denote the mean square of curvature of the trajectory $\mathbf{P}(\tau)$ when parameter $\tau$ varies from 0 to 1 . Using (16) and the derivative chain rule, this can be computed as a function of parameters $\lambda_{0}$ and $\lambda_{1}$ as follows:

$$
k\left(\lambda_{0}, \lambda_{1}\right) \hat{=} \frac{1}{L} \cdot \int_{0}^{L} K^{2}(s) \cdot d s=\frac{1}{L^{4}} \cdot \int_{0}^{1}\left(\frac{d^{2} \mathbf{P}(\tau)}{d \tau^{2}}\right)^{T} \cdot \frac{d^{2} \mathbf{P}(\tau)}{d \tau^{2}} \cdot d \tau
$$

Parameters $\lambda_{0}$ and $\lambda_{1}$ will be computed so that the mean square of curvature is minimized subject to the constraint that the horizontal trajectory length is $L_{h}$.

$$
\min \left(k\left(\lambda_{0}, \lambda_{1}\right)\right) \text { s.t. } l\left(\lambda_{0}, \lambda_{1}\right)=L_{h}
$$

Horizontal trajectory length is $L_{h}$ is defined from (11) by:

$$
L_{h}=V\left(h_{0}\right) \cdot\left(T-t_{d}\right)+L_{d} \cdot \cos \left(\gamma_{d}\right)
$$

Solution of this optimization problem can be found through classical theory of minima and maxima ${ }^{13}$. First, an augmented function $H$ is formed by using a Lagrange multiplier $\mu$ :

$$
H\left(\lambda_{0}, \lambda_{1}\right)=k\left(\lambda_{0}, \lambda_{1}\right)+\mu \cdot\left(l\left(\lambda_{0}, \lambda_{1}\right)-L_{h}\right)
$$

Then, stationary points of $H$ are required to satisfy:

$$
\left\{\begin{array}{l}
\left\{\begin{array}{l}
\frac{\partial H}{\partial \lambda_{0}}=\frac{\partial k\left(\lambda_{0}, \lambda_{1}\right)}{\partial \lambda_{0}}+\mu \cdot \frac{\partial l\left(\lambda_{0}, \lambda_{1}\right)}{\partial \lambda_{0}}=0 \\
\frac{\partial H}{\partial \lambda_{1}}=\frac{\partial k\left(\lambda_{0}, \lambda_{1}\right)}{\partial \lambda_{1}}+\mu \cdot \frac{\partial l\left(\lambda_{0}, \lambda_{1}\right)}{\partial \lambda_{1}}=0 \\
l\left(\lambda_{0}, \lambda_{1}\right)-L_{h}=0
\end{array}\right.
\end{array}\right.
$$

The two first equations can be combined in order to be solved in the Lagrange multiplier $\mu$. Thus, the preceding set of three equations can be reduced to a set of two equations where Lagrange multiplier $\mu$ do not appear:

$$
\left\{\begin{array}{l}
\frac{\partial l\left(\lambda_{0}, \lambda_{1}\right)}{\partial \lambda_{0}} \cdot \frac{\partial k\left(\lambda_{0}, \lambda_{1}\right)}{\partial \lambda_{1}}-\frac{\partial l\left(\lambda_{0}, \lambda_{1}\right)}{\partial \lambda_{1}} \cdot \frac{\partial k\left(\lambda_{0}, \lambda_{1}\right)}{\partial \lambda_{0}}=0 \\
l\left(\lambda_{0}, \lambda_{1}\right)-L_{h}=0
\end{array}\right.
$$

We get from (33) and (35) the following expressions for the partial derivatives:

$$
\begin{aligned}
& \frac{\partial l\left(\lambda_{0}, \lambda_{1}\right)}{\partial \lambda_{0}}=\int_{0}^{1} \frac{\left(\frac{\partial}{\partial \lambda_{0}}\left(\frac{d \mathbf{P}(\tau)}{d \tau}\right)\right)^{T} \cdot \frac{d \mathbf{P}(\tau)}{d \tau}}{\sqrt{\frac{d \mathbf{P}(\tau)^{T}}{d \tau} \cdot \frac{d \mathbf{P}(\tau)}{d \tau}}} \cdot d \tau \quad \text { and } \quad \frac{\partial l\left(\lambda_{0}, \lambda_{1}\right)}{\partial \lambda_{1}}=\int_{0}^{1} \frac{\left(\frac{\partial}{\partial \lambda_{1}}\left(\frac{d \mathbf{P}(\tau)}{d \tau}\right)\right)^{T} \cdot \frac{d \mathbf{P}(\tau)}{d \tau}}{\sqrt{\frac{d \mathbf{P}(\tau)^{T}}{d \tau} \cdot \frac{d \mathbf{P}(\tau)}{d \tau}}} \cdot d \tau \\
& \frac{\partial k\left(\lambda_{0}, \lambda_{1}\right)}{\partial \lambda_{0}}=\frac{2}{L^{4}} \cdot \int_{0}^{1}\left(\frac{\partial}{\partial \lambda_{0}}\left(\frac{d^{2} \mathbf{P}(\tau)}{d \tau^{2}}\right)\right)^{T} \cdot \frac{d^{2} \mathbf{P}(\tau)}{d \tau^{2}} \cdot d \tau \quad \text { and } \quad \frac{\partial k\left(\lambda_{0}, \lambda_{1}\right)}{\partial \lambda_{1}}=\frac{2}{L^{4}} \cdot \int_{0}^{1}\left(\frac{\partial}{\partial \lambda_{1}}\left(\frac{d^{2} \mathbf{P}(\tau)}{d \tau^{2}}\right)\right)^{T} \cdot \frac{d^{2} \mathbf{P}(\tau)}{d \tau^{2}} \cdot d \tau
\end{aligned}
$$

In addition, we have from (27) and (29) the following expressions:

$$
\begin{aligned}
& \frac{\partial}{\partial \lambda_{0}}\left(\frac{d}{d \tau}\left[\begin{array}{l}
x(\tau) \\
y(\tau)
\end{array}\right]\right)=3 \cdot(\tau-1) \cdot(3 \tau-1) \cdot \frac{\partial}{\partial \lambda_{0}} \mathbf{P}_{1 / 3}(\tau)+3 \cdot \tau \cdot(1-\tau)^{2} \cdot L \cdot\left[\begin{array}{l}
\cos \left(\psi_{0}\right) \\
\sin \left(\psi_{0}\right)
\end{array}\right] \\
& \Leftrightarrow \frac{\partial}{\partial \lambda_{0}}\left(\frac{d}{d \tau}\left[\begin{array}{l}
x(\tau) \\
y(\tau)
\end{array}\right]\right)=6 \cdot \tau \cdot(\tau-1) \cdot(2 \tau-1) \cdot L \cdot\left[\begin{array}{l}
\cos \left(\psi_{0}\right) \\
\sin \left(\psi_{0}\right)
\end{array}\right]
\end{aligned}
$$

And

$$
\begin{aligned}
& \frac{\partial}{\partial \lambda_{1}}\left(\frac{d}{d \tau}\left[\begin{array}{l}
x(\tau) \\
y(\tau)
\end{array}\right]\right)=3 \cdot \tau \cdot(2-3 \tau) \cdot \frac{\partial}{\partial \lambda_{1}} \mathbf{P}_{2 / 3}(\tau)+3 \cdot \tau^{2} \cdot(1-\tau) \cdot L \cdot\left[\begin{array}{c}
\cos \left(\psi_{1}\right) \\
\sin \left(\psi_{1}\right)
\end{array}\right] \\
& \Leftrightarrow \frac{\partial}{\partial \lambda_{1}}\left(\frac{d}{d \tau}\left[\begin{array}{l}
x(\tau) \\
y(\tau)
\end{array}\right]\right)=-6 \cdot \tau \cdot(\tau-1) \cdot(2 \tau-1) \cdot L \cdot\left[\begin{array}{c}
\cos \left(\psi_{1}\right) \\
\sin \left(\psi_{1}\right)
\end{array}\right]
\end{aligned}
$$

Similarly, we get from (27) and (30) the following expressions: 
And

$$
\begin{aligned}
& \frac{\partial}{\partial \lambda_{0}}\left(\frac{d^{2}}{d \tau^{2}}\left[\begin{array}{l}
x(\tau) \\
y(\tau)
\end{array}\right]\right)=3 \cdot(6 \tau-4) \cdot \frac{\partial}{\partial \lambda_{0}} \mathbf{P}_{1 / 3}(\tau)+3 \cdot(\tau-1) \cdot(3 \tau-1) \cdot L \cdot\left[\begin{array}{l}
\cos \left(\psi_{0}\right) \\
\sin \left(\psi_{0}\right)
\end{array}\right] \\
& \Leftrightarrow \frac{\partial}{\partial \lambda_{0}}\left(\frac{d^{2}}{d \tau^{2}}\left[\begin{array}{l}
x(\tau) \\
y(\tau)
\end{array}\right]\right)=3 \cdot\left(9 \tau^{2}-8 \tau+1\right) \cdot L \cdot\left[\begin{array}{l}
\cos \left(\psi_{0}\right) \\
\sin \left(\psi_{0}\right)
\end{array}\right]
\end{aligned}
$$

As far as the solving the set equations (40) with expressions (41) - (46) is quite involved, numerical computation is used to solve this set of equations of the form:

$$
\left\{\begin{array}{l}
f_{1}\left(\lambda_{0}, \lambda_{1}\right)=0 \\
f_{2}\left(\lambda_{0}, \lambda_{1}\right)=0
\end{array}\right.
$$

\section{Procedure to build aircraft guidance cues}

Knowing the desired duration of the trajectory, denoted $T$, as well as the initial and final equivalent airspeed and flight level of the aircraft (see Fig. 2), the methodology to build the reference trajectory to be flown by the aircraft is the following:

- Firstly, compute the desired length of the trajectory, $L$, as well as the duration of the descent phase, denoted $t_{d}$, using relationships (8), (10), (11) and (12). The value of duration $t_{d}$ sets the start of descent instant and the longitudinal profile of the aircraft. As far as aircraft guidance cues are concerned, the scheduling of the longitudinal autopilot modes is the following: select flight level $h_{0}$ and airspeed $V_{e}$; then, when the start of descent point is reached, select flight path angle $\gamma_{d}$ and airspeed $V_{e l}$.

- Secondly, define the initial and final positions of the air based coordinates of the aircraft (see Fig. 3 ) from the ground based endpoints constraints $\left(x_{g}(0), y_{g}(0), x_{g}(T)\right.$ and $\left.y_{g}(T)\right)$ by using $(15)$ and the knowledge of wind speed and direction from where wind is blowing, $V_{w}$ and $\psi_{w}$ respectively. In addition, use (13) and speed triangle inversion ${ }^{14}$ to set the initial and final headings, $\psi_{0}$ and $\psi_{1}$ respectively, of the air based reference trajectory from the constraints of initial and final ground based orientation $\chi$ :

$$
\left\{\begin{aligned}
\mathbf{P}_{0 / 3} & =\left[\begin{array}{l}
x_{g}(0) \\
y_{g}(0)
\end{array}\right] \\
\mathbf{P}_{3 / 3} & =\left[\begin{array}{l}
x(T) \\
y(T)
\end{array}\right]=\left[\begin{array}{l}
x_{g}(T)-V_{w} \cdot T \cdot \cos \left(\psi_{w}-\pi\right) \\
y_{g}(T)-V_{w} \cdot T \cdot \sin \left(\psi_{w}-\pi\right)
\end{array}\right] \\
& \psi=\chi-\arcsin \left(\frac{V_{w} \cdot \sin \left(\chi-\psi_{w}\right)}{V \cdot \cos (\gamma)}\right)
\end{aligned}\right.
$$

- Finally solve the parameter optimization problem described in section III-C and compute the lateral reference trajectory $[x(\tau), y(\tau)]^{T}$. As far as guidance cues are concerned, reference heading $\psi_{r}$ is computed from (19) as:

$$
\psi_{r}(\tau)=\arctan \left(\frac{d y(\tau) / d \tau}{d x(\tau) / d \tau}\right)
$$

In order to visualize the ground path $\left[x_{g}(\tau), y_{g}(\tau)\right]^{T}$, the following differential equations (see (14) and (18)) have to be solved, knowing that the airspeed profile $V_{e}(t)$ is known as soon as descent duration $t_{d}$ is computed:

$$
\left\{\begin{array}{ll}
\frac{d}{d t} \tau(t)=\frac{V_{e}(t)}{L \cdot \sqrt{\sigma(h)}} & \text { with }
\end{array} \begin{array}{l}
\tau(0)=0 \\
h(0)=h_{0}
\end{array}\right.
$$

Then, as far as the bijective relationship between parameter $\tau$ is known as a function of time $t$, the ground path is simply obtained by integrating (13): 


$$
\left\{\begin{array}{l}
x_{g}(\tau)=x(\tau)+L \cdot W \cdot \cos \left(\psi_{w}-\pi\right) \cdot \int_{0}^{\tau} \frac{d u}{V(u)}=x(\tau)+W \cdot t(\tau) \cdot \cos \left(\psi_{w}-\pi\right) \\
y_{g}(\tau)=y(\tau)+L \cdot W \cdot \sin \left(\psi_{w}-\pi\right) \cdot \int_{0}^{\tau} \frac{d u}{V(u)}=y(\tau)+W \cdot t(\tau) \cdot \sin \left(\psi_{w}-\pi\right)
\end{array}\right.
$$

As far as the flyability of the reference trajectory is concerned, the reference bank angle $\varphi_{r}$ can be evaluated by:

$$
V \cdot \frac{d \psi(t)}{d t}=g \cdot \tan \left(\varphi_{r}\right) \Rightarrow\left\{\begin{array}{l}
\varphi_{r}=\arctan \left(\frac{V^{2}}{g \cdot L} \cdot \frac{d \psi_{r}(\tau)}{d \tau}\right) \\
\frac{d \psi_{r}(\tau)}{d \tau}=\frac{y^{\prime \prime}(\tau) \cdot x^{\prime}(\tau)-y^{\prime}(\tau) \cdot x^{\prime \prime}(\tau)}{\left(x^{\prime}(\tau)\right)^{2}+\left(y^{\prime}(\tau)\right)^{2}}
\end{array}\right.
$$

\section{Illustrative examples}

The scenario which is used hereafter is built around real life meter fixes of an air navigation procedure to reach Paris Charles De Gaulle airport in France, runway 09R. We assume that an Airbus A330-300 is asked for a 4D operation during its initial approach. More precisely, we will assume that the aircraft is at FL100 with an equivalent airspeed of $250 \mathrm{kts}$ and a course of 36 degrees when she overflies meter fix SUBOX. She is then required to achieve the Initial Fix at $3000 \mathrm{ft}$ with a course of 87 degrees and airspeed of $170 \mathrm{kts}$. Assuming that meter fix is at the origin of the frame, the case study scenario is illustrated on the following figure:

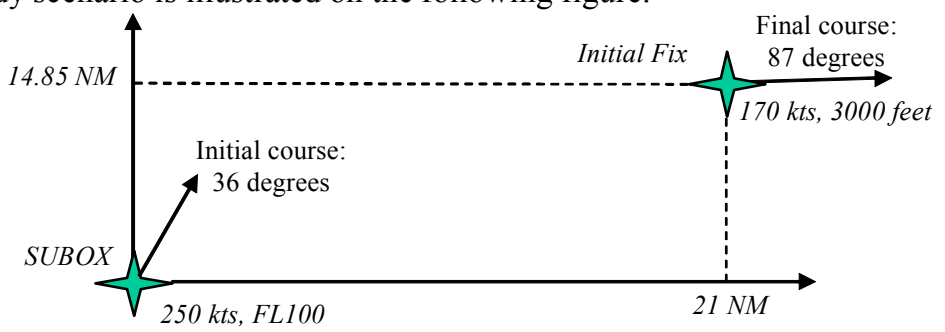

Fig. 4: case study scenario

Flight path angle $\gamma_{d}$ has been set to -3 degrees, whereas deceleration time $t_{s}$ from 250 kts to 170 kts has been set to $80 \mathrm{sec}$ (which corresponds to a deceleration of $-1 \mathrm{kts}$ per second). For this flight profile, the duration of the descent phase, $t_{d}$, has been found to be $404 \mathrm{sec}$ (see (10)).

In the following table, four scenarios are presented: the inputs are the requested duration of the trajectory, denoted $T$, the wind speed $V_{w}$ and the direction from where the wind is blowing, $\psi_{w}$; the outputs are the parameters which enables to set the modified Bezier curves, namely the trajectory length $L$ with respect to the airframe as well as parameters $\lambda_{0}$ and $\lambda_{1}$ :

\begin{tabular}{|c|c|c|c|}
\hline Scenario & $\begin{array}{c}\text { Requested duration } T \\
\text { Wind speed } V_{w} \text { and direction } \psi_{w}\end{array}$ & $\begin{array}{c}\text { Trajectory length with respect to the } \\
\text { airframe } L\end{array}$ & Parameters $\lambda_{0}$ and $\lambda_{1}$ \\
\hline 1 & $\begin{array}{c}T=510 \mathrm{sec} \\
V_{w}=0\end{array}$ & $30.3 \mathrm{NM}$ & $\begin{array}{c}\lambda_{0}=0.323625 \\
\lambda_{1}=1.127037\end{array}$ \\
\hline 2 & $T=600 \mathrm{sec}$ & & $\begin{array}{c}\lambda_{0}=0.403293 \\
\lambda_{1}=1.786681\end{array}$ \\
\hline 3 & $V_{w}=0$ & $37.6 \mathrm{NM}$ & $\lambda_{0}=0.011309$ \\
& $T=510 \mathrm{sec}$ & $30.3 \mathrm{NM}$ & $\lambda_{1}=-0.098885$ \\
\hline 4 & $V_{w}=30 \mathrm{kts}, \psi_{w}=90$ degrees & $37.6 \mathrm{NM}$ & $\lambda_{0}=0.291503$ \\
& $T=600 \mathrm{sec}$ & & $\lambda_{1}=1.382552$ \\
\hline
\end{tabular}

Fig. 5: scenario definition and parameters of the modified Bezier curves

The following figures illustrate the ground path achieved for each scenario. This is the kind of path that air traffic controllers may see on their working positions: 


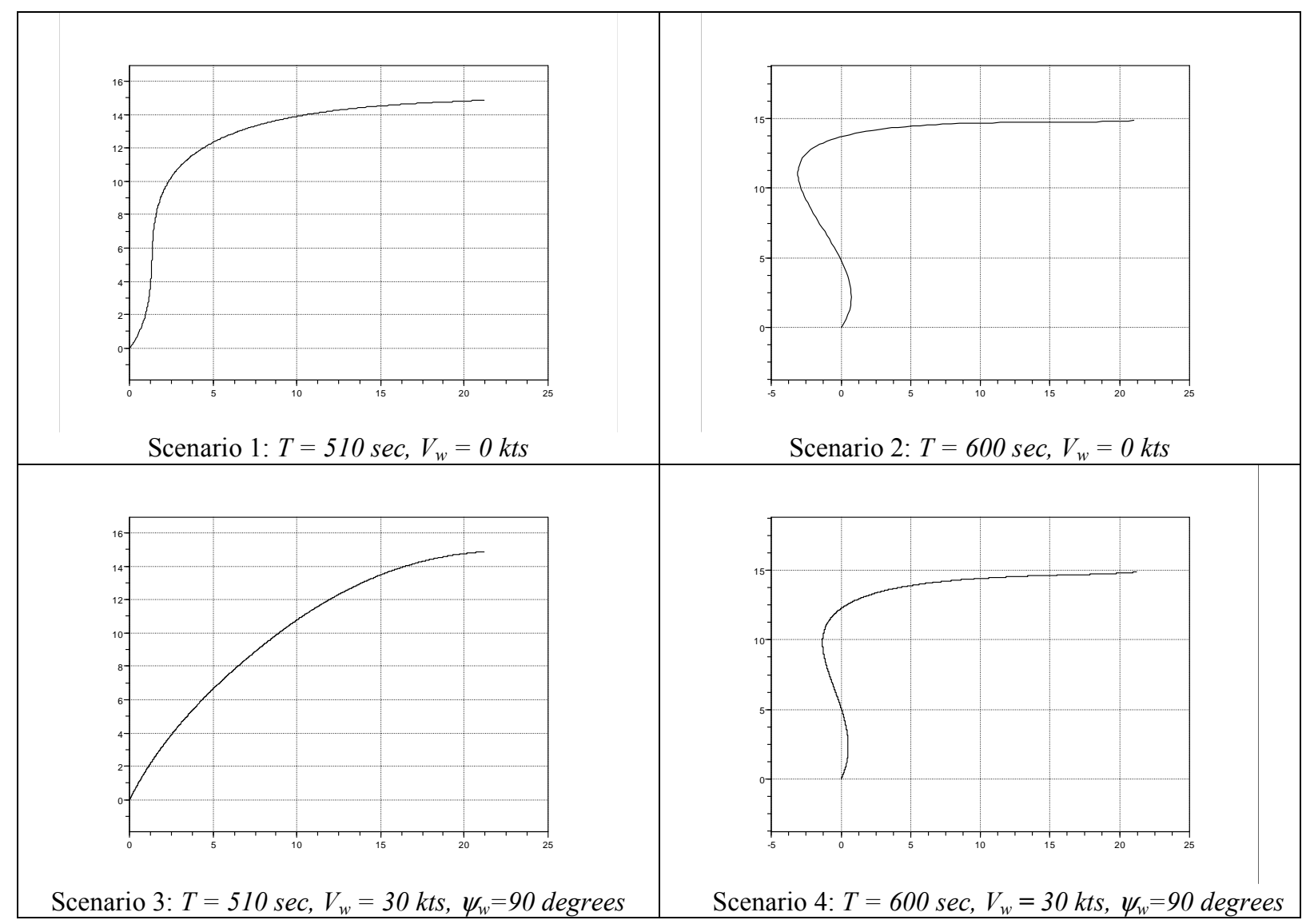

Fig. 6: ground path, Euclidian coordinates in NM

It can be seen from the previous figures that, compared to reference trajectories based on straight lines and circle arcs, which are usual in the aviation community, the proposed approach allows for smooth continuous control cues and avoids the scheduling between straight lines and circle arcs segments.

\section{Conclusion}

In this paper, the design of a new FMS function dedicated to reference path definition for 4D operation has been considered. This new function aims at achieving a specified delay between commercial aircraft at a specified meter fix. This envisioned new capability onboard commercial aircraft provides new perspectives to potentially increase air traffic control efficiency. It could be seen as the airborne counterpart of the ground based arrival manager (AMAN).

The reference path definition is based on modified Bezier curves to generate a path with length and endpoint constraints. The modified Bezier curve is parameterized with a linear function of the arc length, which allows designing the reference trajectory without considering airspeed constraints.

Compared to reference trajectories based on straight lines and circle arcs, which are usual in the aviation community, the proposed approach allows for smooth control cues and avoids the scheduling between straight lines and circle arcs segments.

Simulation results illustrate the effectiveness of the proposed design. Nevertheless, the proposed design is quite demanding in terms of (off line) computational efforts, and improvements may be needed to incorporate it in new FMS functionalities. The tracking of the reference trajectory has also to be addressed. Finally, additional studies in terms of operational scenarios are needed in order to refine and further validate the proposed design.

\section{Acknowledgments}

The authors wish to thank Philippe Louyot, Christelle Pianetti and Jean-Marc Loscos for helpful inputs and comments. 


\section{References}

1 ICAO, Air Traffic Services, Annex 11 to the Convention on International Civil Aviation, Thirteenth Edition, July 2001

2 The Eleventh ICAO Air Navigation Conference, Airborne Separation Assistance System (ASAS), WP64 Item 1.2, Montreal, 22 September to 3 October 2003, http:/www.icao.int/icao/en/anb/meetings/anconf11/index.html

3 SESAR Consortium, The ATM Target Concept - D3, DLM-0612-001-02-00a, 2007, http://www.sesar-consortium.aero/deliv3.php

4 Yamamo M., Iwamura M., Mohri A., Quasic-Time-Optimal Motion Planning of Mobile Platforms in the Presence of Obstacles, International Conference on Robotics and Automation, pp. 739-744 (1999)

5 Fraichard Th., Ahuactzin J. M., Smooth Path Planning for Cars, IEEE International Conference On Robotics and Automation May, pp. 21-26 (2001)

6 Lamiraux F., Kavraki L. E., Planning paths for elastic objects under manipulation constraints, International Journal of Robotics Research, vol. 20, no. 3, pp. 188-208 (2001)

7 Hagelauer, Mora-Camino, Evaluation of Practical Solutions for On Board Aircraft Fourth Dimensional Guidance, Journal of Guidance, Control and Dynamics, Vol 20(5), pp.1052-1054, 1997

8 Bertsekas, Dynamic Programming and Optimal Control, Vol .1, Athena Scientific, Belmont, Massachusetts, 1995

9 Gianazza D., Durand N., Assessment of the 3D-separation of Air Traffic Flows, 6th USA/Europe Seminar on Air Traffic Management Research and Development, Baltimore, June 2005

10 Eshelby, M.E., Aircraft performance: Theory and Practice, AIAA Education Series, 2000

11 Eurocontrol, User Manual For the Base of Aircraft Data (BADA), Revision 3.1, EEC Note No. 10/04, ACE-CE2 (http://www.eurocontrol.int/eec/public/standard_page/proj_BADA.html)

12 Puechmorel S., Delahaye D., 4D Trajectories: a Functional data perspective, $26^{\text {th }}$ DASC conference, Dallas, October 2007

13 Pierre D.A., Optimization Theory With Applications, Dover, 1986

14 Miquel T., Description of an aircraft model for ASAS assessments, DSNA/DTI NT07-619, 2007 